\title{
LUMINOUS SUPERSOFT X-RAY SOURCES
}

\author{
S. RAPPAPORT
}

Department of Physics and Center for Space Research MIT, Cambridge, MA 02139, U.S.A.

AND

R. DI STEFANO

Harvard-Smithsonian Center for Astrophysics

60 Garden St., Cambridge, MA 02138, U.S.A.

\section{Introduction}

Supersoft X-ray sources exhibit spectra that are remarkably steep, in that the ratio of low-to-high energy $X$ rays is much larger than is characteristic of the spectra associated with the previously known classes of luminous $\mathrm{X}$-ray sources. The first supersoft sources were discovered during a survey of the Large Magellanic Cloud with the EINSTEIN Observatory (Long et al. 1981). The all-sky $\mathrm{X}$-ray survey carried out with ROSAT has now established that luminous supersoft $\mathrm{X}$-ray sources constitute a distinct astronomical class (see, e.g., Hasinger 1994). A number of the identified optical counterparts of the supersoft X-ray sources exhibit blue continua with emission lines of $\mathrm{H}$ and He II (Smale et al. 1988; Pakull et al. 1988; Cowley et al. 1990), which are characteristic of accretion disks. The X-ray emission of some sources is steady, while others exhibit significant time variability. Table 1 briefly summarizes what is known thus far about the numbers and characteristics of supersoft X-ray sources (see Hasinger 1994, and references therein).

In Section 2 we focus on a particular model for supersoft sources, one in which the observed luminosity is generated by the nuclear burning of matter that is accreting onto the surface of a white dwarf. We summarize the results of an investigation in which the total population of sources consistent with this model was calculated. Section 3 describes a nearly model-independent theoretical investigation in which we utilized the observed numbers and characteristics of supersoft sources to compute the 
TABLE 1. Supersoft X-Ray Sources: Properties and Statistics

\begin{tabular}{lll}
\hline Range of Properties & Numbers & Identifications \\
\hline$k T \sim 15-55 \mathrm{eV}$ & M31: $\sim 15$ & Binaries (0.5 $<P_{\text {orb }}<3.5$ days): 4 \\
$L \sim 10^{37}-10^{38} \mathrm{ergs} \mathrm{s}^{-1}$ & Galaxy $\sim 6$ & Symbiotic Novae: 3 \\
& LMC $\sim 6$ & Classical Nova: 1 \\
& SMC $\sim 4$ & Planetary Nebula $: 1$ \\
& & X-Ray Pulsar: 1 \\
& & Globular Cluster Source $($ low $L): 1$ \\
\hline
\end{tabular}

${ }^{*}$ We note that this source also has a hard X-ray component.

size of the underlying population of these sources. In Section 4 we continue along a model-independent line of inquiry and sketch several investigations of the ionization nebulae which may be associated with supersoft sources. Section 5 is a summary and a look forward.

\section{Population Synthesis for the Steady Nuclear Burning Model}

Proposed models for the supersoft X-ray sources include nuclear burning on the surface of a white dwarf (Van den Heuvel et al. 1992; Rappaport, Di Stefano \& Smith 1994 [hereafter RDS]), and accreting black holes (Cowley et al. 1990) or neutron stars (Kylafis \& Xilouris 1993; Hughes 1994). In this section, we focus almost exclusively on the nuclear burning model since (1) it is the most quantitative and leads to specific predictions, and (2) the preponderance of evidence points toward a majority of the systems thus far identified having a white dwarf as the X-ray emitting object. Qualitatively, the model we consider invokes steady nuclear burning of accreted matter on the surface of $\mathrm{a} \sim 1 \mathrm{M}_{\odot}$ white dwarf with accretion rates of between $\sim 1$ and $510^{-7} \mathrm{M}_{\odot} \mathrm{yr}^{-1}$; such rates are required to sustain the luminosity (Van den Heuvel et al. 1992; RDS and references therein). In the case of supersoft X-ray sources that are in binary systems with orbital periods in the range of 0.5-3 days, the companion star is expected to be a main-sequence or subgiant of mass $\sim 1.3-2.7 \mathrm{M}_{\odot}$.

The high mass-transfer rates are a natural consequence of unstable mass transfer, on a thermal time scale, via Roche lobe overflow from the more massive donor star to a less massive accreting white dwarf. In the case of supersoft $\mathrm{X}$-ray sources that are associated with symbiotic novae, the mass transfer may be driven by the expansion of a low-mass giant companion as it ascends the giant branch.

An evolutionary scenario for the formation of supersoft $\mathrm{X}$-ray sources with orbital periods near a day is shown in Fig. 1 (which has been adapted 
from Van den Heuvel 1994).

We have carried out a population synthesis calculation of supersoft $\mathrm{X}$ ray sources that are formed via the scenario described above (RDS). We used Monte Carlo techniques to generate the initial binary system, including the primary mass, the mass ratio, and the orbital period. A range of distributions for each of these system parameters was tested. The evolution of each binary was followed through a series of phases (several of which are depicted in Fig. 1) to determine which binaries evolved into systems consisting of a white dwarf accreting matter within the requisite range of rates from a low-mass companion. When a "successful" system was obtained, we recorded the properties of that system.

When properly normalized to the absolute birth rate of stars in the Galaxy, our results yield the number of supersoft X-ray sources, with orbital periods near a day, that should be active in the Galaxy at the present epoch. The results are shown in Fig. 2 in the form of normalized histograms of the predicted properties of (a subset of) the luminous supersoft $\mathrm{X}$-ray sources.

The calculated distributions of properties of supersoft X-ray sources shown in Fig. 2 were for a set of "nominal" input model parameters and assumptions (see RDS). For these assumptions (the "standard" model of RDS), the total number of expected supersoft sources in the Galaxy at the current epoch is about 1000 . We can also scale to the blue magnitudes of other galaxies in the local group to estimate their population of supersoft $\mathrm{X}$-ray sources (see Di Stefano \& Rappaport 1994). Such a procedure yields $\sim 2500,100$, and 30 sources for M31, the LMC, and the SMC, respectively. We have also repeated the above population synthesis calculations for a wide range of input parameters and assumptions (see RDS). The range of values for the predicted numbers of supersoft $\mathrm{X}$-ray sources for the Milky Way, M31, the LMC, and the SMC are summarized in Table 2.

\section{Estimating the Underlying Population of Supersoft Sources}

Radiation at the wavelengths emitted by supersoft sources is readily absorbed by the interstellar medium. It is therefore clear that, whatever the physical nature of the sources (and it is unlikely that any single model will apply to all supersoft sources), ROSAT has not been able to detect a large fraction of the supersoft source population. The question we ask then is: how can we use the relatively small numbers of observed sources to derive an estimate of the true underlying population of presently active sources?

The approach that we have taken (DiStefano \& Rappaport 1994) is to use Monte Carlo techniques to "seed", with a population of sources, each of the galaxies in which supersoft sources have been detected. We then model the gas distribution of each galactic system in order to be able to compute 


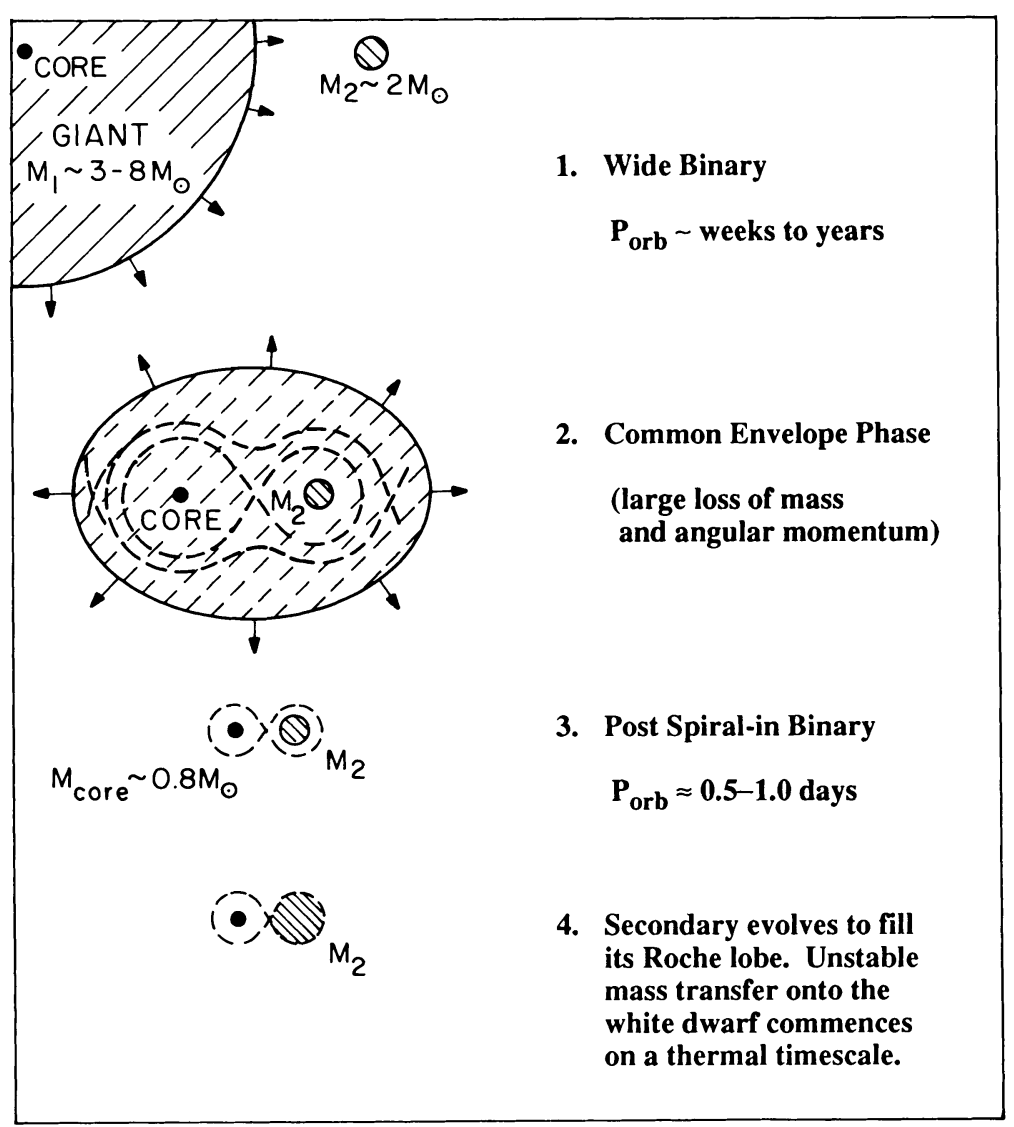

Figure 1. Evolutionary scenario for the formation of a particular class of supersoft X-ray sources. The initial (primordial) binary consists of two main-sequence stars where the primary has a mass in the range of $\sim 2-8 \mathrm{M}_{\odot}$, the secondary has a mass of $>0.8 \mathrm{M}_{\odot}$, and the orbital period is weeks to years. If the primary overfills its Roche lobe while ascending the giant branch, the result will likely be a common-envelope stage (see, e.g., Sparks \& Stecher 1974; Paczyński 1976; Taam, Bodenheimer \& Ostriker 1978; Meyer \& Meyer-Hofmeister 1979; Livio \& Soker 1988; Webbink 1992), leading to the ejection of the giant's envelope. The resultant post-spiral-in binary may consist of a white dwarf (the core of the giant) and a low-mass main-sequence star with an orbital period of about a day. After some time has elapsed, either the orbit or the main-sequence star will evolve so that mass transfer onto the white dwarf will commence. If the donor star is more massive than the accreting white dwarf, the mass transfer will tend to be unstable. As long as the donor star is not sufficiently evolved, the mass transfer will be unstable on a thermal, rather than a dynamical timescale (Paczyński 1965, 1967; Kippenhahn, Kohl \& Weigert 1967; Webbink 1979, 1985, 1992; De Kool 1992). It is important to understand whether the matter so accreted can undergo stable (rather than explosive) nuclear burning. This question has been studied by a number of authors (Paczyński 1970; Sion, Acierno \& Tomcszyk 1979; Taam 1980; Nomoto 1982; Iben 1982; Sion \& Starrfield 1986; Livio, Prialnik \& Regev 1989; Prialnik \& Kovetz 1994). Although the range of mass transfer rates consistent with steady nuclear burning depends on the mass of the white dwarf, typical values are in the range of $\sim(1-5) 10^{-7} \mathrm{M}_{\odot} \mathrm{yr}^{-1}$. 

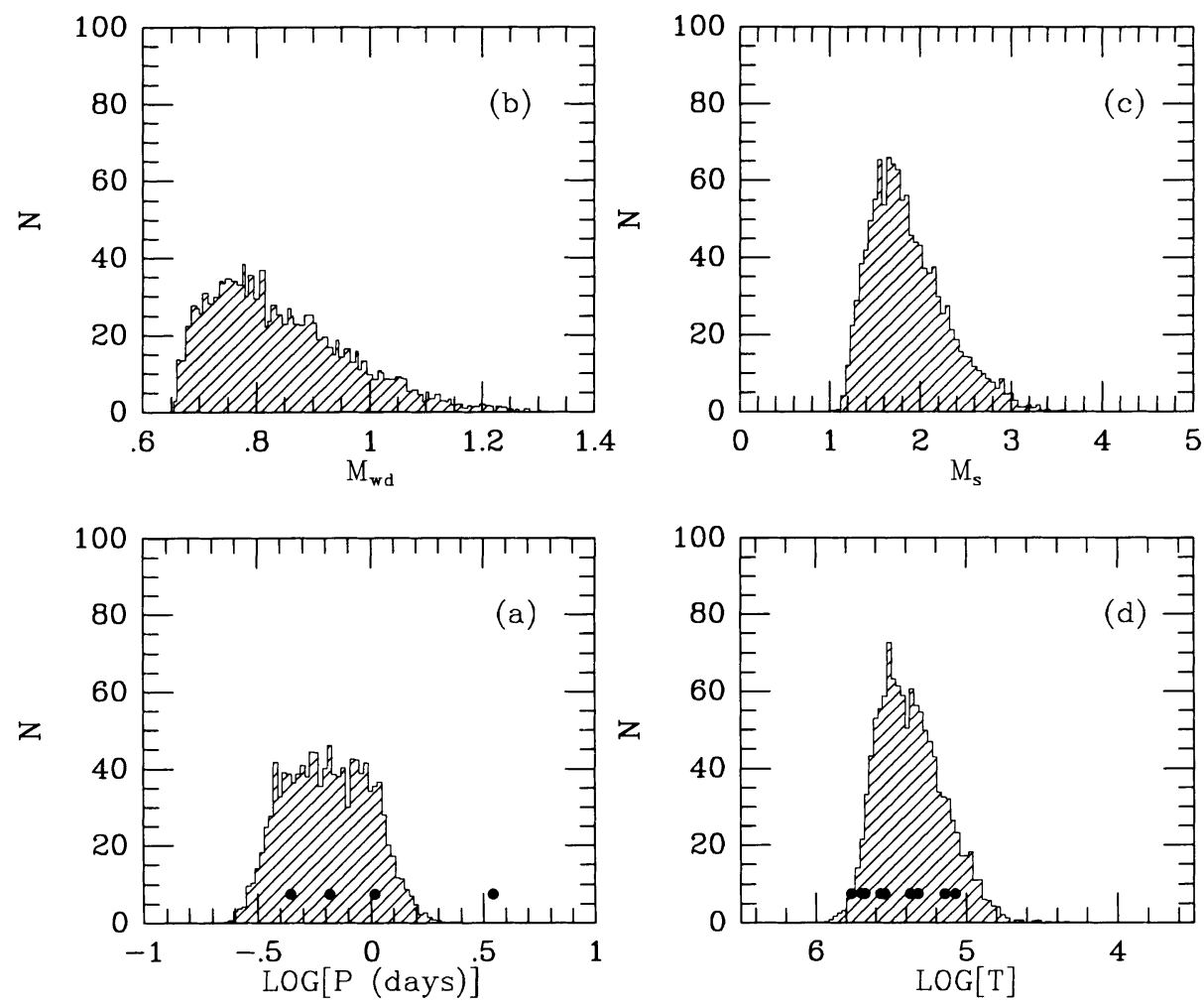

Figure 2. Panels (a) through (d) show the expected distributions of $\boldsymbol{P}_{\text {orb }}$, white dwarf and donor star mass, and effective temperature, $T_{\text {eff }}$, respectively, as derived in the population synthesis study of RDS. The measured values of $T_{\text {eff }}$ for 12 observed systems are superposed on the calculated distribution in (d), while the measured values of $P_{\text {orb }}$ for 4 observed systems are superposed on the calculated distribution in (a). In each case the expected distribution is in good agreement with the observational results. The skew in the measured values of $T_{\text {eff }}$ toward the high end of the distribution is almost certainly an observational selection effect. (See Section 3.) The distribution of white-dwarf and donor star masses indicate most probable values in the ranges of $0.65-1.1 \mathrm{M}_{\odot}$ and $1.5-2.5 \mathrm{M}_{\odot}$, respectively. 
the column density encountered by the radiation from each source as it travels toward Earth. Using the PIMMS software (Mukai 1993), we then compute the number of counts that would be recorded by ROSAT and, depending on the ROSAT observation time for that portion of the sky, we determine whether or not ROSAT would have detected the seeded source.

TABLE 2. Derived Numbers of Supersoft X-Ray Sources

\begin{tabular}{lll}
\hline & Population Synthesis & Inferred from Observation \\
\hline M31 & $400-6000$ & $800-5000$ \\
Milky Way & $100-1500$ & $400-3000$ \\
LMC & $20-300$ & $15-60$ \\
SMC & $5-60$ & $10-40$ \\
\hline
\end{tabular}

In this way, we have been able to compute the fraction, $f$, of all supersoft sources that would have been detected by ROSAT, and hence to use the size of the observed population to infer the size of the total population of presently active sources. The results are summarized in the right-hand column of Table 2, above. In comparing the population estimates (Table 2) with the numbers of sources observed (Table 1 ), one can see that the fraction of sources that ROSAT detects is different for different host galaxies in the Local Group. The value of $1 / f$ lies between $\sim 2.5$ and 10 for the Magellanic Clouds, but ranges from 100 to 750 for the Milky Way, where the majority of sources would have to be detected through the large column densities associated with the disk. Distance plays a significant role in decreasing the number of sources detectable in M31. However, whatever the differences in the values of $f$ from galaxy to galaxy within the Local Group, in all cases $f$ is small.

The fact that there might be a large population of supersoft sources not detectable with ROSAT motivates a search for other ways to detect and study supersoft sources. A promising line of research is the study of the ionization nebulae which may be associated with at least some supersoft sources. These nebulae are described in the next section.

\section{Ionization Nebulae Surrounding Supersoft X-Ray Sources}

\subsection{THEORY}

Regardless of what the nature of luminous supersoft X-ray sources is, it is clear that they emit copious quantities of highly ionizing photons in the range $20 \mathrm{eV}$ to $200 \mathrm{eV}$. Thus, we expect that there will be ionization nebulae surrounding these sources (Rappaport et al. 1994). 
We have carried out calculations of the ionization and temperature structure of the nebulae expected to surround supersoft X-ray sources, as well as their optical line fluxes (Rappaport et al. 1994). The models for ionization nebulae were calculated with the XSTAR code (Kallman \& Krolik 1993) which uses the computational methods described by Kallman \& McCray (1982). The models consist of a spherical gas cloud with a point source of continuum radiation at the center. The input parameters include the source spectrum, the gas composition and density, the initial ionization parameter (which determines the initial radius), and the column density of the cloud (which determines the outer radius). Construction of a model consists of the simultaneous determination of the state of the gas and the radiation field as a function of distance from the source. The state of the gas at each radius follows from the assumption of a stationary local balance between heating and cooling and between ionization and recombination. The calculated characteristic radius of the ionized regions ( $\sim 6 \mathrm{pc}$ for an ISM density of $10 \mathrm{~cm}^{-3}$ ) agrees with what one obtains from a rough estimate based on the Strömgren-sphere formula.

An important question to answer is whether it is possible to distinguish the nebulae associated with supersoft sources (hereafter called supersoft nebulae) from the nebulae associated with other astrophysical objects. In this regard, geometrical considerations are useful. For example, the size of supersoft nebulae can be used to distinguish them from planetary nebulae which typically have radii that are an order of magnitude smaller. It may also be possible to use geometrical considerations to distinguish supersoft nebulae from HII regions. The reason for this is that it is expected that the boundary of supersoft nebulae will be less sharply defined than that of classical $\mathrm{HII}$ regions since there is substantial power in photons with energies $(100-300 \mathrm{eV})$ that are able to reach moderate and even arbitrarily large distances from the source.

Moreover, it may also be possible to draw spectral distinctions between supersoft nebulae and other astrophysical nebulae. For example, Rappaport et al. (1994) showed that these ionization nebulae should be very bright in [O III $] \lambda 5007$, with the ratio of $[\mathrm{O} \mathrm{III]}] / \mathrm{H} \beta$ in the range of $\sim 12-26$ for a solar composition. In the inner parts of these nebulae, this ratio may be larger than for any other known astrophysical object (see, e.g., Osterbrock 1989).

The detection of an ionization nebula around a supersoft X-ray source should lead to a better understanding of both the source luminosity (since, in a sense, the interstellar medium acts as a giant bolometer) and the properties of the surrounding interstellar medium itself. The development of efficient techniques for searching for such ionization nebulae would represent a new means of discovering supersoft $\mathrm{X}$-ray sources using ground-based observations (i.e., at optical wavelengths). This could lead to the optical 
discovery of many of the supersoft X-ray sources that are undetectable in the UV and soft X-ray bands because of severe attenuation by interstellar gas (Di Stefano \& Rappaport 1994).

\subsection{OBSERVATIONS}

Recently, a comprehensive search for ionized gaseous nebulae surrounding 9 known supersoft $\mathrm{X}$-ray sources in the Large and Small Magellanic Clouds was carried out (Remillard, Rappaport \& Macri 1994). (One source, CAL 83, was already known to have an associated nebula [Pakull \& Motch 1989]). Deep images were made using narrow-band filters to isolate the emission lines of $\mathrm{H} \alpha$ and [O III] ( $\lambda 5007)$. In the narrow-band images of Remillard et al. (1994) the CAL 83 nebula is detected out to distances as far as $20 \mathrm{pc}$ from the central source, and the integrated luminosity in each line is of the order of $100 \mathrm{~L}_{\odot}$. If we interpret the observed line luminosities in terms of the model nebula calculations (Rappaport et al. 1994), we conclude that the time-averaged X-ray luminosity of CAL 83 over the past $\sim 310^{4}$ years is $\sim 310^{37} \mathrm{ergs} \mathrm{s}^{-1}$. The bright inner nebula contains $\sim 150 \mathrm{M}_{\odot}$ within $\sim 8 \mathrm{pc}$ of the central source, which clearly indicates that the nebular material is part of the interstellar medium and has not been ejected from the binary system.

In contrast, Remillard et al. (1994) reported no detections of nebulae associated with the other 8 luminous supersoft X-ray sources in the Magellanic Clouds, with upper limits for the [O III] luminosity that are a factor of $\sim 10$ below that for the CAL 83 nebula. For these sources, either the timeaveraged X-ray luminosity of the central source is substantially below that of CAL 83, or the local interstellar medium in the vicinity of these sources is much less dense.

\subsection{IMPLICATIONS}

The association of nebulae with even a subset of all active supersoft sources may have interesting implications. For example, typical supersoft nebula luminosities in the $\lambda 5007$ line of [O III] can be on the order of several times $10^{36} \mathrm{erg} \mathrm{s}^{-1}$. This is comparable to the value of the cut-off luminosity in this line for the planetary nebula luminosity function (PNLF) (see, e.g., Jacoby et al. 1992). We therefore note that these nebulae may possibly be confused with the most luminous planetary nebulae in distant galaxies (Di Stefano, Paerels \& Rappaport 1994). Such confusion would have two potential consequences. First, it might mean that a subset of the nebulae identified through programs designed to study the PNLF may, in fact, be supersoft nebulae. If this subset can be identified, then this would give us a new store of supersoft nebulae to study. Second, such an effect would 
possibly have a small, but systematic influence on the results obtained by using the PNLF to compute intergalactic distances.

\section{Conclusions and Future Directions}

In Sections 2 and 3 we have described investigations which indicate that the underlying population of presently active supersoft sources may be large - on the order of several thousand sources for a galaxy such as M31. Although it will be difficult for observations at X-ray wavelengths to study this population, the ionization nebulae that are likely to be associated with at least a subset of them may provide another way for us to discover and study supersoft sources.

An important implication of the white dwarf accretor model for supersoft sources is the possibility that some of these systems will accrete enough mass to explode as type Ia supernovae. The computations in RDS assess the number of potential supernovae across a range of model parameters, including the uncertain retention of accreted matter by the white dwarf for different mass accretion rates. For at least one set of plausible model parameters, the computed rate of type Ia supernovae was comparable to the observed rate (Cappellaro et al. 1993). A more detailed investigation is underway.

In short, supersoft sources seem likely to be related to a number of interesting astrophysical phenomena. The study of these objects should provide continuing insights for a number of years to come.

Acknowledgements. This work was supported in part by the National Aeronautics and Space Administration under contract NAS 5-29298 and grant NAGW-1545.

\section{References}

Cappellaro, E. et al. 1993, A\&A 273, 383

Cowley, A.P., Schmidtke, P.C., Crampton, D. \& Hutchings, J.B. 1990, ApJ 350, 288

de Kool, M. 1992, A\&A 261, 188

Di Stefano, R. \& Rappaport, S. 1994, (in press)

Di Stefano, R., Paerels, F. \& Rappaport, S. 1994, (in preparation)

Hasinger, G. 1994, in Evolution of X-Ray Binaries, S.S. Holt \& C.S. Day (Eds.), AIP

Conf. Proc. 308, Amer. Inst. Phys. (New York), p. 611

Hughes, J.P. 1994, ApJ 427, L25

Iben Jr., I. 1982, ApJ 259, 244

Jacoby, G.H. et al. 1992, PASP 104, 599

Kallman, T.R. \& McCray, R.A. 1982, ApJS 50, 263

Kallman, T.R. \& Krolik, J.H. 1993, (preprint)

Kippenhahn, R., Kohl, K. \& Weigert, A. 1967, Zeits. für Astrophys. 66, 58

Kylafis, N.D. \& Xilouris, E. 1993, A\&A 278, L43 
Livio, M. \& Soker, N. 1988, ApJ 329, 764

Livio, M., Prialnik, D. \& Regev, O. 1989, ApJ 341, 299

Long, K.S., Helfand, D.J. \& Grabelsky, D.A. 1981, ApJ 248, 925

Meyer, F. \& Meyer-Hofmeister, E. 1979, A\&A 78, 167

Mukai, K. 1993, (private communication)

Nomoto, K. 1982, ApJ 253, 798

Osterbrock, D.E. 1989, Astrophysics of Gaseous Nebulae Univ. Sci. Books (Mill Valley)

Paczyński, B. 1965, Acta Astr. 15, 89

Paczyński, B. 1967, Acta Astr. 17, 193

Paczyński, B. 1970, Acta Astr. 20, 287

Paczyński, B. 1976, in Structure and Evolution of Close Binary Systems, IAU Symp. 73, P. Eggleton, S. Mitton \& J. Whelan (Eds.), Reidel, p. 75

Pakull, M.W. \& Motch, C. 1989, in ESO Workshop on Extranuclear Activity in Galaxies, E.J.A. Meurs \& R.A.E. Fosbury (Eds.), p. 285

Pakull, M.W., Beuermann, K., Van der Klis, M. \& Van Paradijs, J. 1988, A\&A 203, L27

Prialnik, D. \& Kovetz, A. 1994, (preprint)

Rappaport, S., Di Stefano, R. \& Smith, J.D. 1994, ApJ 426, 492 (RDS)

Rappaport, S., Chiang, E., Kallman, T. \& Malina, R. 1994, ApJ 431, 237

Remillard R., Rappaport, S. \& Macri, L. 1994, ApJ (in press)

Sion, E.M. \& Starrfield, S.G. 1986, ApJ 303, 130

Sion, E.M., Acierno, M.J. \& Tomcszyk, S. 1979, ApJ 230, 832

Smale, A.P. et al. 1988, MNRAS 233, 51

Sparks, W.M. \& Stecher, T.P. 1974, ApJ 188, 149

Taam, R.E. 1980, ApJ 242, 749

Taam, R.E., Bodenheimer, P. \& Ostriker, J.P. 1978, ApJ 222, 269

Van den Heuvel, E.P.J. 1994, (private communication)

Van den Heuvel, E.P.J., Bhattacharya, D., Nomoto, K. \& Rappaport, S.A. 1992, A\&A 262, 97

Webbink, R. 1979, in White Dwarfs and Variable Degenerate Stars, IAU Colloquium 53, H. Van Horn \& V. Weidemann (Eds.), University of Rochester Press, p. 426

Webbink, R.F. 1985, in Interacting Binary Stars, J.E. Pringle \& R.A. Wade (Eds.), Cambridge Univ. Press, p. 39

Webbink, R. 1992, in X-Ray Binaries and Recycled Pulsars, E.P.J. Van den Heuvel \& S. Rappaport (Eds.), Kluwer Academic Publishers, p. 269 